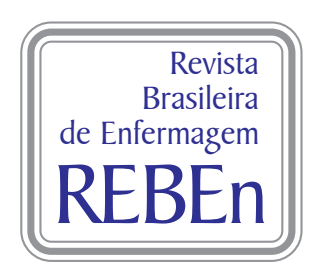

\title{
Conhecimento do cuidador de crianças com hidrocefalia
}

\author{
Knowledge of caregivers of children with hydrocephalus \\ Conocimiento del cuidador de niños con hidrocefalia
}

\section{Débora Moura da Paixão Oliveira', Carlos Umberto Pereira', Záira Moura da Paixão Freitas'}

'Universidade Federal de Sergipe. Departamento de Enfermagem. Aracaju, SE

\author{
Submissão: 14/01/2010 Aprovação: 16/05/2010
}

\begin{abstract}
RESUMO
Pouco se discute sobre conhecimentos dos cuidadores de crianças com hidrocefalia. Este estudo buscou identificar as fontes de informação dos cuidadores e verificar seus conhecimentos sobre hidrocefalia. Foram entrevistados 54 cuidadores com idade entre 18 a 52 anos no período de novembro de 2007 a agosto de 2008. O gênero do cuidador foi predominantemente feminino. Vinte e nove cuidadoras $(53,7 \%)$ aprenderam a cuidar sozinhas. Apenas Quatro cuidadoras $(16,0 \%)$ receberam orientações na alta hospitalar. Vinte e nove $(53,7 \%)$ conhecem a definição e 1 I $(20,4 \%)$ conhecem as causas da hidrocefalia. Verificou-se maior conhecimento entre cuidadoras com mais de oito anos de estudo. A escolaridade teve relação significativa para maior conhecimento, entretanto as cuidadoras possuem pouco conhecimento sobre aspectos importantes da hidrocefalia.
\end{abstract}

Descritores: Hidrocefalia; Cuidado; Conhecimento; Família.

\begin{abstract}
Knowledge of caregivers of children with hydrocephalus is not well assessed. This study identifies the fonts of information to assume activities how to care; to verify caregivers' knowledge about hydrocephalus. Fifty-four caregivers answered the interview from november 2007 to august 2008, with absolute predominance of females. Twenty-nine (53.7\%) learned to take care by themselves. Only four caregivers (16.0\%) received information at the hospital. 29 (53.7\%) regarding the definition, 11 (20.4\%) the cause of hydrocephalus. It was observed that caregivers with eight years of study had bigger knowledge. The education level of the caregiver had a positive correlation to on increased knowledge about hydrocephalus, but the caregivers have little concerns about important hydrocephalus' aspects.
\end{abstract}

Key words: Hydrocephalus; Care; Knowledge; Family.

\section{RESUMEM}

Poco se discute sobre los conocimientos de los cuidadores de niños con hidrocefalia. Este estudio identificó las fuentes de información de los cuidadores y verificó sus conocimientos acerca de la hidrocefalia. Se entrevistó 54 cuidadores con 18 a 52 años desde noviembre de 2007 a agosto de 2008. El género del cuidador fue predominantemente femenino. Veinte y nove cuidadoras $(53,7 \%)$ aprenderán a cuidar solas. Apenas cuatro cuidadoras (16,0\%) tuvieran orientaciones a la salida del hospital. Veinte y nove $(53,7 \%)$ conocen la definición y 1 I (20,4\%) conocen las causas da hidrocefalia. Se observó mayor conocimiento entre cuidadoras con más de ocho años de estudio. La escolaridad tuve relación significativa para mayor conocimiento, todavía las cuidadoras posee poco conocimiento acerca de aspectos importantes da hidrocefalia.

Descriptores: Hidrocefalia; Cuidado; Conocimiento; Familia.

\footnotetext{
AUTOR CORRESPONDENTE Débora Moura da Paixão Oliveira. Universidade Federal de Sergipe. Av. Ivo Prado, 612. São José. CEP 490 I5-070.

Aracaju, SE. E-mail: debora_aju@yahoo.com.br
} 


\section{INTRODUÇÃO}

A hidrocefalia congênita e os Quadros de malformações do sistema nervoso central são as mais frequentes situações clínicas da neurocirurgia infantil ${ }^{(1)}$. A criança com hidrocefalia passa por diversos procedimentos Que diferem da rotina de uma criança saudável. Estes procedimentos acabam fazendo parte da rotina de suas famílias ${ }^{(2)}$.

O tratamento de eleição da hidrocefalia é cirúrgico e consiste em derivações lieuórica com interposição de válvulas unidirecionais para a drenagem do Líquor Cefalo RaQuidiano (LCR) do ventrículo lateral para o peritônio ou átrio esQuerdo do coração ${ }^{(3-9)}$.

A criança com hidrocefalia necessita cuidados específicos, geralmente visam instituir medidas de prevenção de complicações pós-operatórias, cuidados com a pele para prevenção de úlceras por pressão na cabeça, manutenção da hidratação e nutrição, bem como, aplicação de medidas de conforto(10-1).

O nível da gravidade das manifestações clínicas apresentados pela criança irá determinar a intensidade de cuidados Que deverão ser destinados a ela ${ }^{(10-15)}$. Alguns cuidados são específicos da equipe de enfermagem, outros, podem ser realizados no domicílio pelo cuidador ${ }^{(1 I)}$.

É comum presenciar familiares cuidando de suas crianças contando com seus próprios conhecimentos e criatividade. A literatura afirma Que essas famílias cuidam baseadas em seus referenciais, crenças, costumes e recursos. Esses referenciais são geralmente, adeuiridos informalmente, baseados na cultura popular, nem sempre compatíveis com a cultura de cuidado da equipe de saúde ${ }^{(11-14)}$.

Este estudo teve como objetivo identificar as fontes de informação de preparo para assumir os cuidados e verificar o conhecimento dos cuidadores sobre aspectos importantes da hidrocefalia.

\section{MÉTODO}

Tratou-se de um estudo descritivo-exploratório. A pesquisa foi desenvolvida com 54 cuidadores de crianças com hidrocefalia congênita atendidas no Ambulatório de Neurocirurgia do Hospital Universitário da Universidade Federal de Sergipe em Aracaju-Brasil. A amostra foi acidental, não probabilística.

A coleta de dados foi realizada no período de novembro de 2007 a agosto de 2008, utilizando-se a técnica de entrevista padronizada.

Neste estudo foi considerado como cuidador familiar principal, o cuidador exclusivamente familiar, não remunerado, responsável pelo cuidado domiciliar, e Que permanece a maior parte do tempo com a criança.
O projeto foi aprovado pelo Comitê de Ética da Universidade Federal de Sergipe com número de identificação CAAE0126.0.107.000-07. Os cuidadores aceitaram participar do estudo e assinaram o Termo de Consentimento Livre e Esclarecido, garantindo os aspectos éticos previstos na Portaria 196/96 do Conselho Nacional de Saúde do Ministério da Saúde.

Para análise estatística foram utilizados os testes Qui-Quadrado de Pearson e Exato de Fisher com a ajuda do programa SPSS (Statistical Package for Social Sciences) versão 15.0.

\section{RESULTADOS}

Cinquenta e Quatro cuidadores foram entrevistados. A idade do cuidador variou de 18 anos a 52 anos (média 27,3 \pm 7 anos). O gênero dos cuidadores foi predominantemente feminino $N=54$ $(\mathrm{P}<0,000 \mathrm{I})$.

Em relação ao grau de parentesco houve predominância significativa de mães $(\mathrm{P}<0,0001)$, sendo 53 (98,14\%) mãe biológica e uma avó.

No Que se refere ao grau de instrução $13(24,1 \%)$ cursaram menos de Quatro anos de estudo, $23(42,6 \%)$ cursaram entre Quatro a oito anos e $18(33,3 \%)$ cursaram mais de oito anos de estudo.

Em relação ao preparo para ser cuidador, apenas 25 cuidadoras $(46,3 \%)$ receberam orientações sobre os cuidados com a criança. Dentre estas, a identificação das fontes de informação de preparo para assumir os cuidados mostrou Que 14 cuidadoras $(56,0 \%)$ receberam orientação de médicos, sete $(28 \%)$ receberam orientação de leigos e Quatro cuidadoras $(16,0 \%)$ receberam orientações no momento da alta hospitalar. Chama a atenção Que $29(53,7 \%)$ não receberam informação e aprenderam a cuidar sozinhas.

Em relação aos conhecimentos dos cuidadores, observou-se Que 25 (46,3\%) conhecem a definição de hidrocefalia. Verificou-se entre as 54 cuidadoras, pouco conhecimento das causas da hidrocefalia $\mathrm{N}=11$ (20,4\%). A análise do conhecimento sobre o tratamento mostrou Que $34(63,0 \%)$ sabem a finalidade da realização da cirurgia.

Verificou-se uma proporção significativa $(\mathrm{P}<0,05)$ de maior conhecimento entre cuidadoras com mais de oito anos de estudo (Tabela 1).

Registrou-se maior conhecimento entre aQuelas que realizam cuidados de um a Quatro anos, no entanto não houve relação entre maior conhecimento e tempo de realização dos cuidados (Tabela 2).

\section{DISCUSSÃO}

A caracterização dos cuidadores deste estudo revelou a predominância do gênero feminino, fato este registrado em outras

Tabela 1. Frequência de conhecimentos sobre hidrocefalia em relação à escolaridade do cuidador.

\begin{tabular}{lccccc}
\hline \multirow{2}{*}{ Conhecimentos (Sim) } & & \multicolumn{3}{c}{ Escolaridade (anos de estudo) } & \multirow{2}{*}{$\mathrm{P}$} \\
\cline { 3 - 5 } & & $<$ 4anos & 4 a 8 anos & $>8$ anos & \\
\cline { 3 - 5 } Definição & $25(46,3 \%)$ & $1(4,0 \%)$ & $10(40,0 \%)$ & $14(56,0 \%)$ & $\mathrm{p}<0,0001$ \\
Causas & $11(20,4 \%)$ & $0(0 \%)$ & $4(36,4 \%)$ & $7(63,6 \%)$ & $\mathrm{p}=0,03$ \\
Tratamento & $34(63,0 \%)$ & $3(8,8 \%)$ & $15(44,2 \%)$ & $16(47,5 \%)$ & $\mathrm{p}=0,001$ \\
\hline
\end{tabular}


Tabela 2. Frequência de conhecimentos dos cuidadores sobre hidrocefalia em relação ao tempo de cuidado.

\begin{tabular}{|c|c|c|c|c|}
\hline \multirow{2}{*}{ Conhecimentos } & \multicolumn{3}{|c|}{ Tempo de cuidado } & \multirow[b]{2}{*}{$\mathrm{p}$} \\
\hline & $<$ I ano $(\mathrm{N}=20)$ & I a 4 anos $(N=20)$ & 5 a 10 anos $(N=14)$ & \\
\hline Definição & $9(36,0 \%)$ & $10(40,0 \%)$ & $6(24,0 \%)$ & $p=0,94$ \\
\hline Tratamento & $13(38,2 \%)$ & $14(41,2 \%)$ & $7(20,6 \%)$ & $p=0,51$ \\
\hline
\end{tabular}

fontes na literatura ${ }^{(13,16)}$. Os achados confirmam que há uma provisão feminina no cuidado da família, elas o assumem baseadas em valores Que foram passados de geração em geração.

A análise do grau de parentesco do cuidador demonstrou predominância significativa de mães. Os resultados encontrados demonstram Que a provisão do cuidado está relacionada com o vínculo consanguíneo ${ }^{(16-18)}$. Outros estudos coincidem em afirmar Que a própria mãe acredita Que ninguém está à altura de cuidar $\mathrm{e}$ proteger seu filho ${ }^{(16-19)}$

A média de idade do cuidador verificada em nosso estudo foi de 27,3 \pm 7 anos. A literatura descreve Que mulheres participam como cuidadores muito cedo em virtude da prevalência da hidrocefalia em filhos de mães jovens ${ }^{(20-21)}$.

No Que se refere ao grau de instrução observou-se um bom índice de alfabetização entre as entrevistadas, porém a escolaridade média localiza-se em maior proporção entre cuidadoras Que cursaram Quatro a oito anos de estudo. Os resultados encontrados por outros autores ${ }^{(19,22)}$ também demonstram um padrão de escolaridade mínima, semelhantes a esta casuística.

Outros estudos ${ }^{(23-24)}$ observaram Que o nível de escolaridade foi significativamente associado com altos níveis de conhecimento. Porém, vale ressaltar Que estes estudos são na maioria das vezes realizados em grandes centros urbanos brasileiros ou em países desenvolvidos. A baixa escolaridade observada no estudo pode ser relacionada à escolaridade da população sergipana em geral e à instituição local de pesquisa Que atende as classes sociais menos favorecidas usuárias do Sistema Único de Saúde (SUS).

Algumas pesquisas ${ }^{(25-27)}$ teceram reflexões sobre a importância da atuação do enfermeiro por meio de estratégias de educação em saúde aos cuidadores. Segundo estes autores o enfermeiro, como educador deve oferecer subsídios aos cuidadores. Ao potencializarmos as famílias como cuidadoras, estas poderão servir de multiplicadoras de conhecimentos em sua comunidade, ampliando o alcance da atuação profissional na sociedade ${ }^{(14)}$.

No Que se refere ao preparo para assumir a posição de cuidador, verificou-se, em relação à distribuição das fontes de informações de cuidadores, Que apenas 46,3\% receberam orientações. Os dados assemelham-se aos resultados encontrados em outra publicação, onde apenas $31,6 \%$ dos cuidadores referiram ter sido orientado ${ }^{(28)}$.

Os achados desta pesquisa mostraram que $56,0 \%$ dos cuidadores receberam orientação médica. Os achados corroboram outras pesquisas onde os autores observaram que $43 \%$ a $50 \%$ dos pais de crianças com doença crônica tiveram como principais orientadores médicos e enfermeiras ${ }^{(19,29)}$.

Este estudo enfoca a necessidade do preparo do cuidador para a alta hospitalar, chama-se a atenção Que apenas 16\% receberam orientações no momento da alta hospitalar. Os dados aqui encontrados são semelhantes aos registrados por outros autores ${ }^{(28-}$ ${ }^{29)}$, em suas casuísticas, os cuidadores também informaram Que foram orientados por médicos e poucos por enfermeiros, evidenciando Que durante a internação os enfermeiros têm atuado de maneira reduzida na orientação dos familiares.

É fundamental Que o enfermeiro utilize seus conhecimentos e habilidades pedagógicas na orientação dos familiares estabelecendo uma relação de confiança com a família para informá-la sobre a doença $^{(25-27)}$

Outro estudo realizado no Brasil com pacientes neurocirúrgicos demonstrou Que no momento da alta os profissionais ofereceram às famílias uma grande Quantidade de informações visando capacitálos para realizar o cuidado no domicílio ${ }^{(30)}$. $\mathrm{O}$ Que não foi observado neste estudo. Os autores chamaram a atenção Que a alta de pacientes neurocirúrgicos deve ser planejada pela equipe hospitalar e articulada com a unidade básica de saúde mais próxima da residência do paciente.

Nossa casuística mostrou Que $53,7 \%$ da amostra aprenderam a cuidar sozinhas. Esta informação se confirma com outros estudos, alguns autores observaram Que sem orientação de como proceder com os cuidados e sem muito conhecimento sobre a doença, o cuidador constrói na prática diária o seu conhecimento de forma solitária ${ }^{(19,27)}$.

A análise do conhecimento das cuidadoras verificou pouco conhecimento da definição e causas da hidrocefalia. Verificou-se maior conhecimento entre as Que cursaram mais de oito anos de estudo. Uma investigação realizada na TurQuia com familiares de crianças com hidrocefalia mostrou resultados semelhantes, o nível de instrução dos pais teve um efeito significativo para o conhecimento a respeito da definição e causas da hidrocefalia ${ }^{(29)}$.

Uma revisão de literatura sobre o conhecimento dos pais identificou Que o nível educacional da mãe teve maior valor discriminante em relação ao conhecimento ${ }^{(24)}$. As pesQuisas confirmam a importância da escolaridade materna como uma variável relevante aos conhecimentos sobre desenvolvimento infantil ressaltando a importância da escolaridade das mães como uma variável influente sobre a cognição familiar ${ }^{(23-24)}$.

O conhecimento superficial da definição e das causas da hidrocefalia, observado nesta peşuisa, pode ser consęuência da falta de orientação de profissionais ou do uso de linguagem científica ao falarem com os familiares, dificultando a assimilação do conteúdo das informações. Muitas vezes as orientações recebidas pelos cuidadores denotam grande desinformação, o Que para os autores demonstram falta de compromisso dos profissionais com a continuidade do cuidado(26).

\section{CONCLUSÕES}

Este estudo verificou cuidadoras jovens, com pouca escolaridade e poucos conhecimentos sobre definição e causas da hidrocefalia, prestando cuidados a crianças com hidrocefalia, porém, apesar destas 
considerações e de pouca orientação profissional, foi possível observar Que existe entre as cuidadoras um bom conhecimento sobre o tratamento da hidrocefalia. O conhecimento é maior entre cuidadoras com maior escolaridade, independente do tempo de cuidado.

Os dados sugerem Que o cuidador familiar da criança portadora de hidrocefalia não foi objeto de cuidado do enfermeiro.

\section{REFERÊNCIAS}

1. Paes N. Hidrocefalia. In: Pereira CU. Neurocirurgia pediátrica. Rio de Ianeiro: Revinter; 2000. p. I 1-7.

2. Kulkarni AV, Rabin D, Drake JM. An instrument to measure the health status of children with hydrocephalus: the Hydrocephalus Outcome Questionnaire. I Neurosurg 2004; 10 I (suppl 2): 13440.

3. Chiafery M. Care and management of the child with shunted hydrocephalus. Pediatr Nurs 2006; 32(3): 222-5.

4. Aquino HB, Carelli EF, Borges Neto AG, Pereira CU. Nonfunctional abdominal complications of the distal catheter on the treatment of hydrocephalus: an inflammatory hypothesis? Experience with six cases. Child's Nerv Syst 2006; 22(10): 1225-30.

5. Duhaime AC. Evaluation and management of shunt infections in children with hydrocephalus. Clin Pediatr 2006; 45(8): 705 13.

6. Garton HJ. Cerebrospinal fluid diversion procedures. I Neuroophthalmol 2004; 24(2): 103-5.

7. Heinsbergen I, Rotteveel I, Roeleveld N, Grotenhuis A. Outcome in shunted hydrocephalic children. Eur I Paediatr Neurol 2002; 6(2):99-107.

8. Jucá CEB, Lins Neto A, Oliveira RS, Machado HR. Tratamento de hidrocefalia com derivação ventrículo-peritoneal: análise de 150 casos consecutivos no Hospital das Clínicas de Ribeirão Preto. Acta Cir Bras 2002; 17(Suppl 3):59-63.

9. Kliemann SE, Rosemberg S. Hidrocefalia derivada na infância: um estudo clínico-epidemiológico de 243 observações consecutivas. Are NeuropsiQuiatr 2005; 63(2b): 494-501.

10. Kirk EA, White C, Freeman S. Effects of a nursing education intervention on parents' knowledge of hydrocephalus and shunts. I Neurosci Nurs 1992; 24(2): 99-103.

11. Oliveira DMP. Assistência de enfermagem à criança com hidrocefalia. In: Pereira CU. Neurocirurgia Pediátrica. Rio de Janeiro: Revinter; 2000. p. 57-60.

12. Carneiro TM. Vivenciando o cuidar e o curar como familiar em um hospital. Rev Bras Enferm 2008; 6 I (3):390-4.

13. Collet N, Rocha SMM. Criança hospitalizada: mãe e enfermagem compartilhando o cuidado. Rev Latino-am Enfermagem 2004; 12(2): $191-7$.

14. Gomes GC. Compartilhando o cuidado à criança: refletindo o ser família e construindo um novo modo de cuidar a partir da vivência na internação hospitalar [tese]. Florianópolis: Universidade Federal de Santa Catarina; 2005.

15. Chamberlain MA, Kent RM. The needs of young people with disabilities in transition from pediatric to adult services. Europ Medicophys 2005; 4I(2): 1 II-23.

16. Gallo AM, Hadley EK, Angst DB, Knafl KA, Smith AM. Parents' concerns about issues related to their children's genetic conditions. ISPN 2008; I3(I): 4-I4.
17. Mendes PBMT. Quem é o cuidador? In: Dias ELR, Wanderley IS, Mendes RT, organizadores. Orientações para cuidadores informais na assistência domiciliar. Campinas: Editora da Unicamp; 2002. p. 17-30.

18. Deeken JF, Taylor KL, Mangan P, Yabroff KR, Ingham IM. Care for the caregivers: a review of self-report instruments developed to measure the burden, needs, and Quality of life of informal caregivers. I Pain Symptom Manag 2003; 26(4): 922-53.

19. Santo Pedro K, Marcon SS. Perfil e vivência dos cuidadores informais de doentes crônicos assistidos pelo NEPAAF - Núcleo de estudos, pesquisa, assistência e apoio à família. Online Brazilian I Nurs 2007. Available from: http://www.uff.br/ objnursing.

20. Ludueña MP, Prada EMG. Características clínicas de recién nacidos internados por mielomeningocele en el Hospital del Niños “Dr. Ovidio Aliaga Uría. 1993-2002. Rev Soc Bol Ped 2003; 42(3): 160-5.

21. Cáceres FM, Uscátegui AM, Rojas ID, Bacerra CH, Díaz LA. Registro durante un año de vigilancia activa no selectiva en el Hospital Universitario Ramón Gonzalez Valencia. Medunab 1999; 2(6): 109-14.

22. Ertem IO, Atay G, Dogan DG, Bayhan A, Bingoler BE, Gok CG, et al. Mothers' knowledge of young child development in a developing country. Child Care Health Dev 2007; 33(6): 728-37.

23. Moura MLS, Ribas Jr. RC, Piccinini CA. Conhecimento sobre desenvolvimento infantil em mães primíparas de diferentes centros urbanos do Brasil. Estud Psicol 2004; 9(3):42 I-9.

24. Ribas Ir RC, Moura MLS, Bornstein MH. Socioeconomic status in Brazilian psychological research. Part II: socioeconomic status and parenting knowledge. Estud Psicol 2003; 8(3):385-92.

25. Silva ACL, Badia I. Significado do cuidar da criança portadora de hidrocefalia na percepção do familiar [monografia]. São Carlos: Universidade Federal de São Carlos; 2004.

26. Silva JB, Kirschbaum DIR, Oliveira I. Significado atribuído pelo enfermeiro ao cuidado prestado à criança doente crônica hospitalizada acompanhada de familiar. Rev Gaúcha Enferm 2007; 28(2): 250-9.

27. Souza LM, Wegner W, Gorini MIPC. Educação em saúde: uma estratégia de cuidado ao cuidador leigo. Rev Latino-am Enfermagem 2007; 15(2): 6-9.

28. Pereira APS, Tessarini MM, Pinto MH, Oliveira VDC. Alta hospitalar: visão de um grupo de enfermeiras. Rev Enferm UERJ 2007; 15(1): 40-5.

29. Yilmaz G, Eroahin Y, Turhan T. A Survey in parents of the patients with shunted hydrocephalus. I Neurol Sci Turk 2006; 23(4): 303-6.

30. Knihs NS, Franco SC. A família vivenciando o cuidado do paciente neurocirúrgico: necessidades e expectativas frente a esse cuidado. Ciên Cuid Saúde 2005; 4(2): 139-48. 